Astrophysics of super-massive black hole mergers

This content has been downloaded from IOPscience. Please scroll down to see the full text. 2013 Class. Quantum Grav. 30244007

(http://iopscience.iop.org/0264-9381/30/24/244007)

View the table of contents for this issue, or go to the journal homepage for more

Download details:

IP Address: 128.183.97.246

This content was downloaded on 25/04/2014 at 13:27

Please note that terms and conditions apply. 


\title{
Astrophysics of super-massive black hole mergers
}

\author{
Jeremy D Schnittman \\ NASA Goddard Space Flight Center, Greenbelt, MD 20771, USA \\ E-mail: jeremy.schnittman@nasa.gov
}

Received 12 July 2013, in final form 15 October 2013

Published 29 November 2013

Online at stacks.iop.org/CQG/30/244007

\begin{abstract}
We present here an overview of recent work in the subject of astrophysical manifestations of super-massive black hole (SMBH) mergers. This is a field that has been traditionally driven by theoretical work, but in recent years has also generated a great deal of interest and excitement in the observational astronomy community. In particular, the electromagnetic (EM) counterparts to SMBH mergers provide the means to detect and characterize these highly energetic events at cosmological distances, even in the absence of a spacebased gravitational-wave observatory. In addition to providing a mechanism for observing SMBH mergers, EM counterparts also give important information about the environments in which these remarkable events take place, thus teaching us about the mechanisms through which galaxies form and evolve symbiotically with their central black holes.
\end{abstract}

PACS numbers: 95.30.Sf, 98.54.Cm, 98.62.Js, 04.30.Tv, 04.80.Nn

(Some figures may appear in colour only in the online journal)

\section{Introduction}

Following numerical relativity's annus mirabilis of 2006, a deluge of work has explored the astrophysical manifestations of black hole mergers, from both the theoretical and observational perspectives. While the field has traditionally been dominated by applications to the direct detection of gravitational waves (GWs), much of the recent focus of numerical simulations has been on predicting potentially observable electromagnetic (EM) signatures ${ }^{1}$. Of course, the greatest science yield will come from coincident detection of both the GW and EM counterpart, giving a myriad of observables such as the black hole mass, spins, redshift, and host environment, all with high precision [29]. Yet even in the absence of a direct GW detection (and this indeed is the likely state of affairs for at least the next decade), the EM signal alone may be sufficiently strong to detect with wide-field time-domain surveys, and also unique enough to identify unambiguously as a super-massive black hole (SMBH) merger.

1 By 'signature', we mean any observable property of a given source that indicates, directly or indirectly, that a SMBH merger has taken place, or will take place within a Hubble time. An important subset of direct signatures includes EM 'counterparts', those sources that are coincident with GW detections. 
In this article, we review the brief history and astrophysical principles that govern the observable signatures of SMBH mergers. To date, the field has largely been driven by theory, but we also provide a summary of the observational techniques and surveys that have been utilized, including recent claims of potential detections of both SMBH binaries and also post-merger recoiling black holes.

While the first public use of the term 'black hole' is generally attributed to John Wheeler in 1967, as early as 1964 Edwin Saltpeter proposed that gas accretion onto SMBHs provided the tremendous energy source necessary to power the highly luminous quasi-stellar objects (quasars) seen in the centers of some galaxies [217]. Even earlier than that, black holes were understood to be formal mathematical solutions to Einstein's field equations [226], although considered by many to be simply mathematical oddities, as opposed to objects that might actually exist in nature (perhaps most famously, Eddington's stubborn opposition to the possibility of astrophysical black holes probably delayed significant progress in their understanding for decades) [257].

In 1969, Lynden-Bell outlined the foundations for black hole accretion as the basis for quasar power [159]. The steady-state thin discs of Shakura and Sunyaev [235], along with the relativistic modifications given by Novikov and Thorne [182], are still used as the standard models for accretion discs today. In the following decade, a combination of theoretical work and multi-wavelength observations led to a richer understanding of the wide variety of accretion phenomena in active galactic nuclei (AGN) [205]. In addition to the well-understood thermal disc emission predicted by Shakura and Sunyaev and Novikov and Thorne [182, 235], numerous non-thermal radiative processes such as synchrotron and inverse-Compton are also clearly present in a large fraction of AGN [70, 186].

Peters and Mathews [196] derived the leading-order gravitational wave emission from two point masses more than a decade before Thorne and Braginsky [256] suggested that one of the most promising sources for such a GW signal would be the collapse and formation of a SMBH, or the (near head-on) collision of two such objects in the center of an active galaxy. In that same paper, Thorne and Braginsky build on earlier work by Estabrook and Wahlquist [75] and explore the prospects for a space-based method for direct detection of these GWs via Doppler tracking of inertial spacecraft. They also attempted to estimate event rates for these generic bursts, and arrived at quite a broad range of possibilities, from $\lesssim 0.01$ to $\gtrsim 50$ events per year, numbers that at least bracket our current best-estimates for SMBH mergers [227].

However it is not apparent that Thorne and Braginsky considered the hierarchical merger of galaxies as the driving force behind these SMBH mergers, a concept that was only just emerging at the time, and was still controversial [188, 189, 245, 259]. Within the galactic merger context, the seminal paper by Begelman, Blandford, and Rees (BBR) [18] outlines the major stages of the SMBH merger: first the nuclear star clusters merge via dynamical friction on the galactic dynamical time $t_{\mathrm{gal}} \sim 10^{8} \mathrm{yr}$; then the SMBHs sink to the center of the new stellar cluster on the stellar dynamical friction time scale $t_{\mathrm{df}} \sim 10^{6} \mathrm{yr}$; the two SMBHs form a binary that is initially only loosely bound, and hardens via scattering with the nuclear stars until the loss cone is depleted; further hardening is limited by the diffusive replenishing of the loss cone, until the binary becomes 'hard', i.e., the binary's orbital velocity is comparable to the local stellar orbital velocity, at which point the evolutionary time scale is $t_{\text {hard }} \sim N_{\text {inf }} t_{\text {df }}$, with $N_{\text {inf }}$ stars within the influence radius. This is typically much longer than the Hubble time, effectively stalling the binary merger before it can reach the point where gravitational radiation begins to dominate the evolution. Since $r_{\text {hard }} \sim 1 \mathrm{pc}$, and GWs do not take over until $r_{\mathrm{GW}} \sim 0.01 \mathrm{pc}$, this loss cone depletion has become known as the 'final parsec problem' [168]. BBR thus propose that there should be a large cosmological population of stalled SMBH binaries with separation of order a parsec, and orbital periods of years to centuries. Yet to date 
not a single binary system with these sub-parsec separations has even been unambiguously identified $^{2}$.

In the decades since BBR, numerous astrophysical mechanisms have been suggested as the solution to the final parsec problem [168]. Yet the very fact that so many different solutions have been proposed and continue to be proposed is indicative of the prevailing opinion that it is still a real impediment to the efficient merger of SMBHs following a galaxy merger. However, the incontrovertible evidence that galaxies regularly undergo minor and major mergers during their lifetimes, coupled with a distinct lack of binary SMBH candidates, strongly suggest that nature has found its own solution to the final parsec problem. Or, as Einstein put it, 'God does not care about mathematical difficulties; He integrates empirically'.

For incontrovertible evidence of a SMBH binary, nothing can compare with the direct detection of GWs from space. The great irony of GW astronomy is that, despite the fact that the peak GW luminosity generated by black hole mergers outshines the entire observable universe, the extremely weak coupling to matter makes both direct and indirect detection exceedingly difficult. For GWs with frequencies less than $\sim 1 \mathrm{~Hz}$, the leading instrumental concept for nearly 25 years now has been a long-baseline laser interferometer with three free-falling test masses housed in drag-free spacecraft [76]. Despite the flurry of recent political and budgetary constraints that have resulted in a number of alternative, less capable designs, we take as our fiducial detector the classic LISA (laser interferometer space antenna) baseline design [150].

For SMBHs with masses of $10^{6} M_{\odot}$ at a redshift of $z=1$, LISA should be able to identify the location of the source on the sky within $\sim 10 \mathrm{deg}^{2}$ a month before merger, and better than $\sim 0.1 \mathrm{deg}^{2}$ with the entire waveform, including merger and ringdown [128, 129, 142-144, 164, 255]. This should almost certainly be sufficient to identify EM counterparts with wide-field surveys such as LSST [1], WFIRST [246], or WFXT [178]. Like the cosmological beacons of gamma-ray bursts and quasars, merging SMBHs can teach us about relativity, high-energy astrophysics, radiation hydrodynamics, dark energy, galaxy formation and evolution, and how they all interact.

A large variety of potential EM signatures have recently been proposed, almost all of which require some significant amount of gas in the near vicinity of the merging black holes [223]. Thus we must begin with the question of whether or not there is any gas present, and if so, what are its properties. Only then can we begin to simulate realistic spectra and light curves, and hope to identify unique observational signatures that will allow us to distinguish these objects from the myriad of other high-energy transients throughout the universe.

\section{Circumbinary discs}

If there is gas present in the vicinity of a SMBH binary, it is likely in the form of an accretion disc, as least at some point in the system's history. Discs are omnipresent in the universe for the simple reason that it is easy to lose energy through dissipative processes, but much more difficult to lose angular momentum. At larger separations, before the SMBHs form a bound binary system, massive gas discs can be quite efficient at bringing the two black holes together [64, 74]. As these massive gas discs are typically self-gravitating, their dynamics can be particularly complicated, and require high-resolution 3D simulations, which will be discussed in more detail in section 3.5.

While the massive discs are important for initially driving the SMBHs toward merger, here we focus on the properties of non-self-gravitating circumbinary accretion discs, which will be

2 A notable exception is the radio galaxy $0402+379$ [210], with a projected separation of 7.3 pc, which would in fact qualify as a hard binary for the inferred total mass of $\sim 7 \times 10^{8} M_{\odot}[211]$. 
responsible for the bulk of the EM emission close to merger. The inner regions of these discs have traditionally employed the same alpha prescription for pressure-viscous stress scaling as in Shakura and Sunyaev [235]. Much of the early work on this subject was applied to protoplanetary discs around binary stars, or stars with massive planets embedded in their surrounding discs. The classical work on this subject is Pringle [200], who considered the evolution of a 1D thin disc with an additional torque term added to the inner disc. This source of angular momentum leads to a net outflow of matter, thus giving these systems their common names of 'excretion' or 'decretion' discs. Pringle considered two inner boundary conditions: one for the inflow velocity $v^{r}\left(R_{\text {in }}\right) \rightarrow 0$ and one for the surface density $\Sigma\left(R_{\text {in }}\right) \rightarrow 0$. For the former case, the torque is applied at a single radius at the inner edge, leading to a surface density profile that increases steadily inwardtoward $R_{\text {in }}$. In the latter case, the torque is applied over a finite region in the inner disc, which leads to a relatively large evacuated gap out to $\gtrsim 6 R_{\text {in }}$. In both cases, the angular momentum is transferred from the binary outward through the gas disc, leading to a shrinking of the binary orbit.

In [8], SPH simulations were utilized to understand in better detail the torquing mechanism between the gas and disc. They find that, in agreement with the linear theory of Goldreich and Tremaine [88], the vast majority of the binary torque is transmitted to the gas through the $(l, m)=(1,2)$ outer Lindblad resonance (for more on resonant excitation of spiral density waves, see [249]). The resonant interaction between the gas and eccentric binary ( $e=0.1$ for the system in [8]) pumps energy and angular momentum into the gas, which gets pulled after the more rapidly rotating interior point mass. This leads to a nearly evacuated disc inside of $r \approx 2 a$, where $a$ is the binary's semi-major axis. The interaction with the circumbinary disc not only removes energy and angular momentum from the binary, but it can also increase its eccentricity, and cause the binary pericenter to precess on a similar timescale, all of which could lead to potentially observable effects in GW observations [6, 212, 213].

In subsequent papers [9, 10], Artymowicz and Lubow expand upon their original work [8] and provide a comprehensive study of the effects of varying the eccentricity, mass ratio, and disc thickness on the behavior of the circumbinary disc and its interaction with the binary. Not surprisingly, they find that the disc truncation radius moves outward with binary eccentricity. Similarly, the mini accretion discs around each of the stars has an outer truncation radius that decreases with binary eccentricity. On the other hand, the location of the inner edge of the circumbinary disc appears to be largely insensitive to the binary mass ratio [9]. For relatively thin, cold discs with aspect ratios $H / R \approx 0.03$, the binary torque is quite effective at preventing accretion, much as in the decretion discs of Pringle [200]. In that case, the gas accretion rate across the inner gap is as much as $10-100 \times$ smaller than that seen in a single disc, but the authors acknowledge that the low resolution of the SPH simulation makes these estimates inconclusive [9].

When increasing the disc thickness to $H / R \approx 0.1$, the gas has a much easier time jumping the gap and streaming onto one of the two stars, typically the smaller one. For $H / R \approx 0.1$, the gas accretion rate is within a factor of two of the single-disc case [10]. The accretion rate across the gap is strongly modulated at the binary orbital period, although the accretion onto the individual masses can be out of phase with each other. The modulated accretion rate suggests a promising avenue for producing a modulated EM signal in the pre-merger phase, and the very fact that a significant amount of gas can in fact cross the gap is important for setting up a potential prompt signal at the time of merger.

To adequately resolve the spiral density waves in a thin disc, 2D grid-based calculations are preferable to the inherently noisy and diffusive SPH methods. Armitage and Natarayan [5] take a hybrid approach to the problem, and use a 2D ZEUS [247] hydrodynamics calculation to normalize the torque term in the 1D radial structure equation. Unlike [8], they find almost 

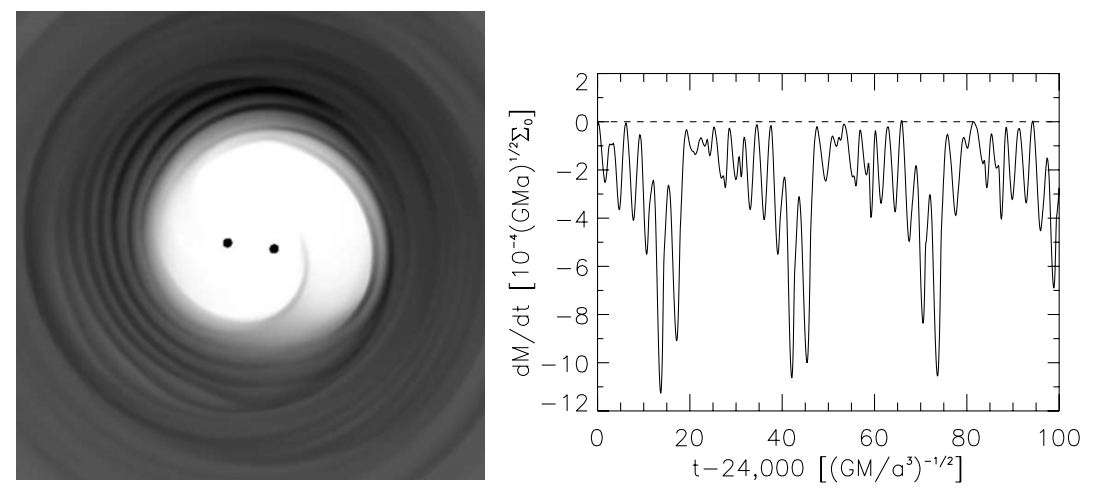

Figure 1. Left: surface density and spiral density wave structure of circumbinary disc with equalmass BHs on a circular orbit, shown after the disc evolved for 4000 binary periods. The dimensions of the box are $x=[-5 a, 5 a]$ and $y=[-5 a, 5 a]$. Right: time-dependent accretion rate across the inner edge of the simulation domain $\left(r_{\text {in }}=a\right)$, normalized by the initial surface density scale $\Sigma_{0}$. (Reproduced from [160]).

no leakage across the gap, even for a moderate $H / R=0.07$. However, they do identify a new effect that is particularly important for binary black holes, as opposed to protoplanetary discs. For a mass ratio of $q \equiv m_{2} / m_{1}=0.01$, when a small accretion disc is formed around the primary, the evolution of the secondary due to gravitational radiation can shrink the binary on such short time scales that it plows into the inner accretion disc, building up gas and increasing the mass accretion rate and thus luminosity immediately preceding merger [5]. If robust, this obviously provides a very promising method for generating bright EM counterparts to SMBH mergers. However, recent 2D simulations by [17] suggest that the gas in the inner disc could actually flow across the gap back to the outer disc, like snow flying over the plow. The reverse of this effect, gas piling up in the outer disc before leaking into the inner disc, has recently been explored by $[131,132]$.

In the context of $\mathrm{T}$ Tau stars, [96, 97] developed a sophisticated simulation tool that combines a polar grid for the outer disc with a Cartesian grid around the binary to best resolve the flow across the gap. They are able to form inner accretion discs around each star, fed by persistent streams from the circumbinary disc. As a test, they compare the inner region to an SPH simulation and find good agreement, but only when the inner discs are artificially fed by some outer source, itself not adequately resolved by the SPH calculation [97]. They also see strong periodic modulation in the accretion rate, due to a relatively large binary eccentricity of $e=0.5$.

MacFadyen and Milosavljevic (MM08) [160] also developed a sophisticated grid-based code including adaptive mesh refinement to resolve the flows at the inner edge of the circumbinary disc in the SMBH binary context. However, they excise the inner region entirely to avoid excessive demands on their resolution around each black hole so are unable to study the behavior of mini accretion discs. They also use an alpha prescription for viscosity and find qualitatively similar results to the earlier work described above: a gap with $R_{\text {in }} \approx 2 a$ due to the $m=2$ outer Lindblad resonance, spiral density waves in an eccentric disc, highly variable and periodic accretion, and accretion across the gap of $\sim 20 \%$ that expected for a single $\mathrm{BH}$ accretion disc with the same mass [160]. The disc surface density as well as the variable accretion rate are shown in figure 1 . Recent work by the same group carried out a systematic study of the effect of mass ratio and found significant accretion across the gap for all values of $q=m_{2} / m_{1}$ between 0.01 and 1 [63]. 
The net result of these calculations seems to be that circumbinary gas discs are a viable mechanism for driving the SMBH binary through the final parsec to the GW-driven phase, and supplying sufficient accretion power to be observable throughout. Thus it is particularly perplexing that no such systems have been observed with any degree of certainty. According to simple alpha-disc theory, there should also be a point in the GW evolution where the binary separation is shrinking at such a prodigious rate that the circumbinary disc cannot keep up with it, and effectively decouples from the binary. At that point, gas should flow inward on the relatively slow timescale corresponding to accretion around a single point mass, and a real gap of evacuated space might form around the SMBHs, which then merge in a near vacuum [174]. However, the quantitative details of how effective circumbinary discs are at driving the system to merger, and providing accretion material along the way, are still open questions, hopefully to be resolved by future simulations (see below, section 3.5).

\section{Numerical simulations}

\subsection{Vacuum numerical relativity}

In the context of EM counterparts, the numerical simulation of two equal-mass, non-spinning black holes in a vacuum is just about the simplest problem imaginable. Yet the inherent nonlinear behavior of Einstein's field equations made this a nearly unsolvable Grand Challenge problem, frustrating generations of relativists from the $3+1$ formulation of Arnowitt, Deser, and Misner in 1962 [7], followed shortly by the first attempt at a numerical relativity (NR) simulation on a computer in 1964 [98], decades of uneven progress, slowed in large part by the limited computer power of the day (but also by important fundamental instabilities in the formulation of the field equations), to the ultimate solution in late 2005, reached independently via the excision method by Pretorius [198] and with the moving puncture method developed simultaneously by the Brownsville and Goddard groups [11, 43]. These initial breakthrough papers all focused on quasi-circular, equal-mass, (nearly) non-spinning binaries, and were followed by a subsequent deluge of papers in 2006 from multiple groups around the world (for a much more thorough review of this colorful story and the many technical challenges overcome by its participants, see [46]).

Here we will review just a few highlights from the recent NR results that are most pertinent to our present subject. For the first 50 years since their original conception, black holes (and general relativity as a whole) were largely relegated to mathematicians as a theoretical curiosity with little possibility of application in astronomy. All this changed in the late 1960s and early 1970s when both stellar-mass and SMBHs were not only observed, but also understood to be critical energy sources and play a major role in the evolution of galaxies and stars [257]. A similar environment was present during the 1990s with regard to binary black holes and GWs. Most believed in their existence, but after decades of false claims and broken promises, the prospect of direct detection of GWs seemed further away than ever. But then in 1999, construction was completed on the two LIGO observatories, and they began taking science data in 2002. At the same time, the space-based LISA concept was formalized with the 'Yellow Book', a report submitted to ESA in 1996, and together with NASA, an international science team was formed in 2001. Astrophysics theory has long been data-driven, but here was a case where large-scale projects were being proposed and even funded based largely on theoretical predictions.

The prospect of real observations and data in turn energized the NR community and provided new motivation to finally solve the binary $\mathrm{BH}$ merger problem. Long-duration, accurate waveforms are necessary for both the detection and characterization of GWs. Generic 
binary sources are fully described by 17 parameters: the BH masses (2), spin vectors (6), binary orbital elements (6), sky position (2), and distance (1). To adequately cover this huge parameter space requires exceedingly clever algorithms and an efficient method for calculating waveforms. Fortunately, most NR studies to date suggest that even the most nonlinear phase of the inspiral and merger process produces a relatively smooth waveform, dominated by the leading quadrupole mode [46]. Additionally, in the early inspiral and late ringdown phases, relatively simple analytic expressions appear to be quite sufficient in matching the waveforms [194]. Even more encouraging is the fact that waveforms from different groups using very different methods agree to a high level of accuracy, thus lending confidence to their value as a description of the real world [13].

In addition to the waveforms, another valuable result from these first merger simulations was the calculation of the mass and spin of the final black hole, demonstrating that the GWs carried away a full $4 \%$ of their initial energy in roughly an orbital time, and leave behind a moderately spinning black hole with $a / M=0.7[11,43]$.

After the initial breakthrough with equal-mass, non-spinning black holes, the remarkably robust 'moving puncture' method was soon applied to a wide variety of systems, including unequal masses [21], eccentric orbits [111], and spinning BHs [44]. As with test particles around Kerr black holes, when the spins are aligned with the orbital angular momentum, the BHs can survive longer before plunging, ultimately producing more GW power and resulting in a larger final spin. This is another critical result for astrophysics, as the spin evolution of SMBHs via mergers and gas accretion episodes is a potentially powerful diagnostic of galaxy evolution [22]. Perhaps the most interesting and unexpected result from the NR bonanza was the first accurate calculation of the gravitational recoil, which will be discussed in more detail in the following section.

In addition to the widespread moving puncture method, the NR group at Cornell/Caltech developed a highly accurate spectral method that is particularly well-suited for long evolutions [41]. Because it converges exponentially with resolution (as opposed to polynomial convergence for finite-difference methods), the spectral method can generate waveforms with dozens of GW cycles, accurate to a small fraction of phase. These long waveforms are particularly useful for matching the late inspiral to post-Newtonian (PN) equations of motion, the traditional tool of choice for GW data analysis for LIGO and LISA (e.g., [4, 25, 56, 127]). The down side of the spectral method has been its relative lack of flexibility, making it very time consuming to set up simulations of new binary configurations, particularly with arbitrary spins. If this problem can be overcome, spectral waveforms will be especially helpful in guiding the development of more robust semi-analytic tools (e.g., the effective-one-body approach of Buonanno [16]) for calculating the inspiral, merger, and ringdown of binary BHs with arbitrary initial conditions [112].

The natural application for long, high-accuracy waveforms is as templates in the matchedfiltering approach to GW data analysis. For LIGO, this is critical to detect most BH mergers, where much of the in-band power will come from the final stages of inspiral and merger. The high signal-to-noise expected from SMBHs with a future space-based interferometer means that most events will probably be detected with high significance even when using a primitive template library $[57,81]$. However, for parameter estimation, high-fidelity waveforms are essential for faithfully reproducing the physical properties of the source. In particular, for spinning BHs, the information contained in the precessing waveform can greatly improve our ability to determine the sky position of the source, and thus improve our prospects for detecting and characterizing any EM counterpart [143, 144, 255]. 


\subsection{Gravitational recoil}

In the general case where there is some asymmetry between the two black holes (e.g., unequal masses or spins), the GW radiation pattern will have a complicated multipole structure. The beating between these different modes leads to a net asymmetry in the momentum flux from the system, ultimately resulting in a recoil or kick imparted on the final merged black hole [220]. This effect has long been anticipated for any GW source [19, 37, 195], but the specific value of the recoil has been notoriously difficult to calculate using traditional analytic means $[24,58,80,203,269]$. Because the vast majority of the recoil is generated during the final merger phase, it is a problem uniquely suited for NR. Indeed, this was one of the first results published in 2006, for the merger of two non-spinning BHs with mass ratio 3:2, giving a kick of $90-100 \mathrm{~km} \mathrm{~s}^{-1}$ [12].

Shortly thereafter, a variety of initial configurations were explored, covering a range of mass ratios [89, 110], aligned spins [109, 138], and precessing spins [45, 258]. Arguably the most exciting result came with the discovery of the 'superkick' configuration, where two equal-mass black holes have equal and opposite spins aligned in the orbital plane, leading to kicks of $>3000 \mathrm{~km} \mathrm{~s}^{-1}[45,90,258]$. If such a situation were realized in nature, the resulting black hole would certainly be ejected from the host galaxy, leaving behind an empty nuclear host [167]. Some of the many other possible ramifications include offset AGN, displaced star clusters, or unusual accretion modes. These and other signatures are discussed in detail below in section 4.

Analogous to the PN waveform matching mentioned above, there has been a good deal of analytic modeling of the kicks calculated by the NR simulations [40, 202, 218, 220]. Simple empirical fits to the NR data are particularly useful for incorporating the effects of recoil into cosmological $N$-body simulations that evolve SMBHs along with merging galaxies $[14,45,157,262]$. While the astrophysical impacts of large kicks are primarily Newtonian in nature (even a kick of $v \sim 3000 \mathrm{~km} \mathrm{~s}^{-1}$ is only $1 \%$ of the speed of light), the underlying causes, while only imperfectly understood, clearly point to strong nonlinear gravitational forces at work [117, 199, 207, 208, 220].

\subsection{Pure electromagnetic fields}

Shortly after the 2006-07 revolution, many groups already began looking for the next big challenge in NR. One logical direction was the inclusion of EM fields in the simulations, solving the coupled Einstein-Maxwell equations throughout a black hole merger. The first to do so was Palenzuela et al [190], who considered an initial condition with zero electric field and a uniform magnetic field surrounding an equal-mass, non-spinning binary a couple orbits before merger. The subsequent evolution generates E-fields twisted around the two BHs, while the B-field remains roughly vertical, although it does experience some amplification (see figure 2).

The EM power from this system was estimated by integrating the radial Poynting flux through a spherical shell at large radius. They found only a modest (30-40\%) increase in EM energy, but there was a clear transient quadrupolar Poynting burst of power coincident with the GW signal, giving one of the first hints of astrophysical EM counterparts from NR simulations. This work was followed up by a more thorough study in [175, 191], which showed that the EM power $L_{\mathrm{EM}}$ scaled like the square of the total $\mathrm{BH}$ spin and proportional to $B^{2}$, as would be expected for a Poynting flux-powered jet [26]. The astrophysical applications of such simplified calculations are admittedly limited, but they are undoubtedly a crucial step along the way to full NR plus matter simulations, and provide useful scaling relations and intuition about the behavior of magnetic fields in dynamic space-time. 

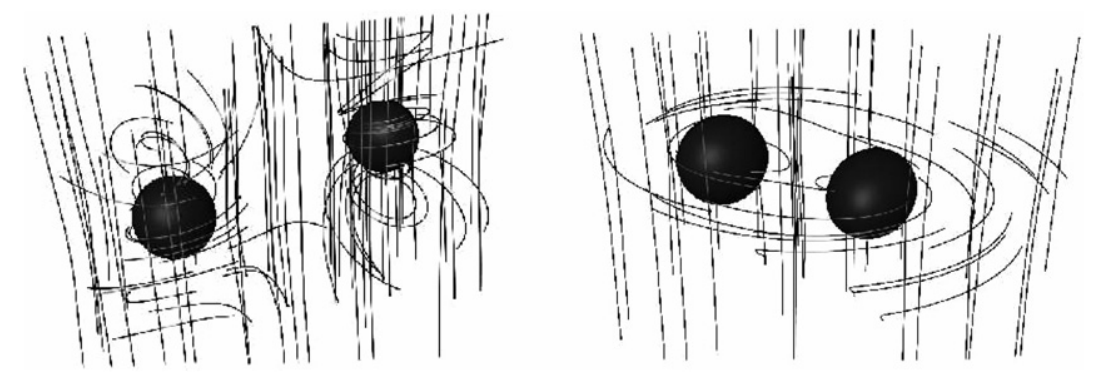

Figure 2. Magnetic and electric field configurations around binary black hole $40 M$ (left) and 20M (right) before merger. The electric fields get twisted around the black holes, while the magnetic fields remain roughly vertical. (Reproduced from [190]).

\subsection{Force-free simulations}

Following these initial vacuum simulations, Palenzuela and collaborators extended their vacuum simulations to include force-free electrodynamics [192,193]. This is an approximation where a tenuous plasma is present, and can generate currents and magnetic fields, but carries no inertia to push those fields around. They found that any moving, spinning black hole can generate Poynting flux and a Blandford-Znajek-type jet [26]. Compared to the vacuum case, force-free simulations of a merging binary predict significant amplification of EM power by a factor of $\sim 10 \times$, coincident with the peak $\mathrm{GW}$ power [193]. For longer simulations run at higher accuracy, $[2,176]$ found an even greater $L_{\mathrm{EM}}$ amplification of $\sim 30 \times$ that of electro-vacuum.

\section{5. $M / H D$ simulations}

As mentioned above in section 2, if there is an appreciable amount of gas around the binary $\mathrm{BH}$, it is likely in the form of a circumbinary disc. This configuration has thus been the subject of most (magneto) hydrodynamical simulations. SPH simulations of discs that are not aligned with the binary orbit show a warped disc that can precess as a rigid body, and generally suffer more gas leakage across the inner gap, modulated at twice the orbital frequency $[106,116$, 145]. In many cases, accretion discs can form around the individual BHs [64, 102].

Massive discs have the ability to drive the binary toward merger on relatively short time scales [55, 64, 74] and also align the BH spins at the same time [32] (although see also $[153,154]$ for a counter result). Retrograde discs may be even more efficient at shrinking the binary [179] and they may also be quite stable [180]. Recent simulations by Roedig et al [213] show that the binary will evolve due not only to torques from the circumbinary disc, but also from transfer of angular momentum via gas streaming onto the two black holes. They find that the binary does shrink, and eccentricity can still be excited, but not necessarily at the rates predicted by classical theory.

Following merger, the circumbinary disc can also undergo significant disruption due to the gravitational recoil, as well as the sudden change in potential energy due to the mass loss from GWs. These effects lead to caustics forming in the perturbed disc, in turn leading to shock heating and potentially both prompt and long-lived EM afterglows [54, 165, 184, 197, $214,215,271,272]$. Any spin alignment would be critically important for both the character of the prompt EM counterpart, as well as the recoil velocity [23, 158].

Due to computational limitations, it is generally only possible to include the last few orbits before merger in a full NR simulation. Since there is no time to allow the system to relax into a quasi-steady state, the specific choice of initial conditions is particularly important for these hydrodynamic merger simulations. Some insight can be gained from Newtonian simulations [240] as well as semi-analytic models [152, 204, 237]. 
If the disc decouples from the binary well before merger, the gas may be quite hot and diffuse around the black holes [105]. In that case, uniform density diffuse gas may be appropriate. In merger simulations by [30, 35, 77], the diffuse gas experiences Bondi-type accretion onto each of the SMBHs, with a bridge of gas connecting the two before merger. Shock heating of the gas could lead to a strong EM counterpart. As a simple estimate for the EM signal, [35] use bremsstrahlung radiation to predict roughly Eddington luminosity peaking in the hard x-ray band.

The first hydrodynamic NR simulations with disc-like initial conditions were carried out by Farris et al [78] by allowing the disc to relax into a quasi-steady state before turning the GR evolution on. They found disc properties qualitatively similar to classical Newtonian results, with a low-density gap threaded by accretion streams at early times, and largely evacuated at late times when the binary decouples from the disc. Due to the low density and high temperatures in the gap, they estimate the EM power will be dominated by synchrotron (peaking in the IR for $M=10^{8} M_{\odot}$ ), and reach Eddington luminosity. An analogous calculation was carried out by Bode et al [31], with somewhat thicker discs, yet they find EM luminosity orders of magnitude smaller, perhaps because of the disc thickness, or perhaps simply because they do not relax the initial disc for as long.

Most recently, circumbinary disc simulations have moved from purely hydrodynamic to ideal magneto-hydrodynamic (MHD; assumes no resistivity in the fluid, which is tied to the magnetic field lines), allowing them to dispense with alpha prescriptions of viscosity and incorporate the true physical mechanism behind angular momentum in accretion discs: magnetic stresses and the magneto-rotational instability [15]. Newtonian MHD simulations of circumbinary discs find large-scale $m=1$ modes growing in the outer disc, modulating the accretion flow across the gap [240]. Similar modes were seen in [181], who used a similar procedure as [78] to construct a quasi-stable state before allowing the binary to merge. They find that the MHD disc is able to follow the inspiraling binary to small separations, showing little evidence for the decoupling predicted by classical disc theory. However, the simulations of Noble et al [181] use a hybrid space-time based on PN theory [83] that breaks down close to merger. Furthermore, while fully relativistic in its MHD treatment, the individual black holes are excised from the simulation due to computational limitations, making it difficult to quantify the properties of an EM counterpart from the inner flow. Farris et al [79] have been able to overcome this issue and put the BHs on the grid with the MHD fluid. They find that the disc decouples at $a \approx 10 M$, followed by a decrease in luminosity before merger, and then an increase as the gap fills in and resumes normal accretion, as in Milosavljevic and Phinney [174].

Giacomazzo et al [87] carried out MHD merger simulations with similar initial conditions to both [191] and [30], with diffuse hot gas threaded by a uniform vertical magnetic field. Unlike in the force-free approximation, the inclusion of significant gas leads to a remarkable amplification of the magnetic field, which is compressed by the accreting fluid. [87] found the B-field increased by of a factor of 100 during merger, corresponding to an increase in synchrotron power by a factor of $10^{4}$, which could easily lead to super-Eddington luminosities from the IR through hard X-ray bands.

The near future promises a self-consistent, integrated picture of binary BH-disc evolution. By combining the various methods described above, we can combine multiple MHD simulations at different scales, using the results from one method as initial conditions for another, and evolve a circumbinary disc from the parsec level through merger and beyond.

\subsection{Radiation transport}

Even with high resolution and perfect knowledge of the initial conditions, the value of the GRMHD simulations is limited by the lack of radiation transport and accurate thermodynamics, 
which have only recently been incorporated into local Newtonian simulations of steady-state accretion discs $[113,114]$. Significant future work will be required to incorporate the radiation transport into a fully relativistic global framework, required not just for accurate modeling of the dynamics, but also for the prediction of direct EM signatures that might be compared directly with observations.

Some recent progress has been made by using the relativistic Monte Carlo ray-tracing code Pandurata as a post-processor for MHD simulations of single accretion discs [224, 225], reproducing soft and hard x-ray spectral signatures in agreement with observations of stellarmass black holes. Applying the same ray-tracing approach to the MHD merger simulations of Giacomazzo et al [87], we can generate light curves and broad-band spectra, ranging from synchrotron emission in the IR up through inverse-Compton peaking in the x-ray. An example of such a spectrum is shown in figure 3, corresponding to super-Eddington luminosity at the peak of the EM and GW emission. Since the simulation in [87] does not include a cooling function, we simply estimate the electron temperature as $100 \mathrm{keV}$, similar to that seen in typical AGN coronas. Future work will explore the effects of radiative cooling within the NR simulations, as well as incorporating the dynamic metric into the ray-tracing analysis.

Of course, the ultimate goal will be to directly incorporate radiation transport as a dynamical force within the GRMHD simulations. Significant progress has been made recently in developing accurate radiation transport algorithms in a fully covariant framework [120, 187, 216], and we look forward to seeing them mature to the point where they can be integrated into dynamic GRMHD codes. In addition to Pandurata, there are a number of other relativistic ray-tracing codes (e.g., [62, 238]), currently based on the Kerr metric, which may also be adopted to the dynamic space-times of merging black holes.

\section{Observations: past, present, and future}

One way to categorize EM signatures is by the physical mechanism responsible for the emission: stars, hot diffuse gas, or circumbinary/accretion discs. In figure 4, we show the diversity of these sources, arranged according the spatial and time scales on which they are likely to occur [223]. Over the course of a typical galaxy merger, we should expect the system to evolve from the upper-left to the lower-center to the upper-right regions of the chart. Sampling over the entire observable universe, the number of objects detected in each source class should be proportional to the product of the lifetime and observable flux of that object.

Note that most of these effects are fundamentally Newtonian, and many are only indirect evidence of SMBH mergers, as opposed to the prompt EM signatures described above. Yet they are also important in understanding the complete history of binary BHs, as they are crucial for estimating the number of sources one might expect at each stage in a black hole's evolution. If, for example, we predict a large number of bright binary quasars with separations around $0.1 \mathrm{pc}$, and find no evidence for them in any wide-field surveys (as has been the case so far, with limited depth and temporal coverage), we would be forced to revise our theoretical models. But if the same rate calculations accurately predict the number of dual AGN with separations of $\sim 1-10 \mathrm{kpc}$, and GW or prompt EM detections are able to confirm the number of actual mergers, then we might infer the lack of binary quasars is due to a lack of observability, as opposed to a lack of existence.

The long-term goal in observing EM signatures will be to eventually fill out a plot like that of figure 4 , determining event rates for each source class, and checking to make sure we can construct a consistent picture of SMBH-galaxy co-evolution. This is indeed an ambitious goal, but one that has met with reasonable success in other fields, such as stellar evolution 


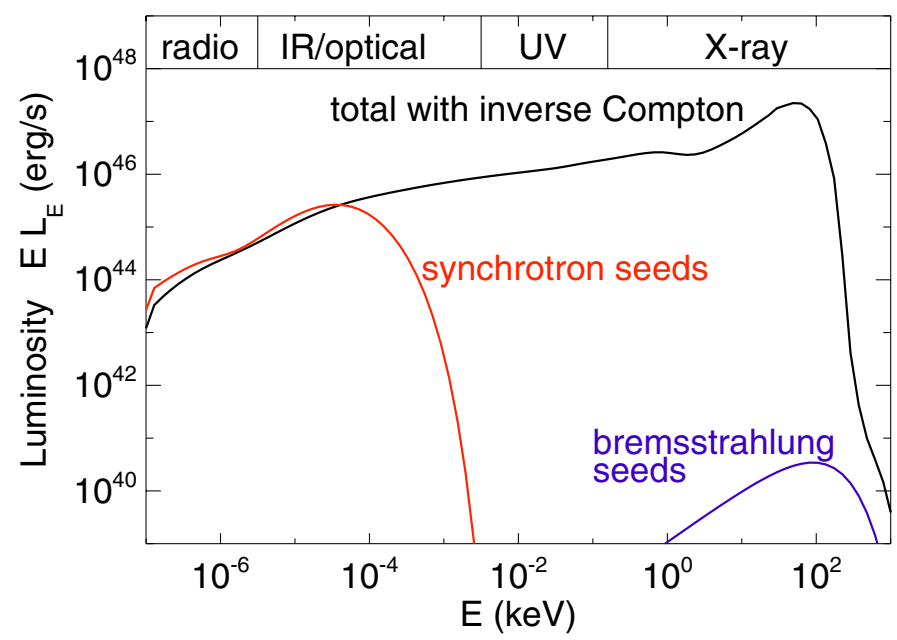

Figure 3. A simplified calculation of the broad-band spectrum produced by the GRMHD merger described in [87], sampled near the peak of gravitational wave emission. Synchrotron and bremsstrahlung seeds from the magnetized plasma are ray-traced with Pandurata [225]. InverseCompton scattering off hot electrons in a diffuse corona gives a power-law spectrum with cut-off around $k T_{e}$. The total mass is $10^{7} M_{\odot}$ and the gas has $T_{e}=100 \mathrm{keV}$ and optical depth of order unity.

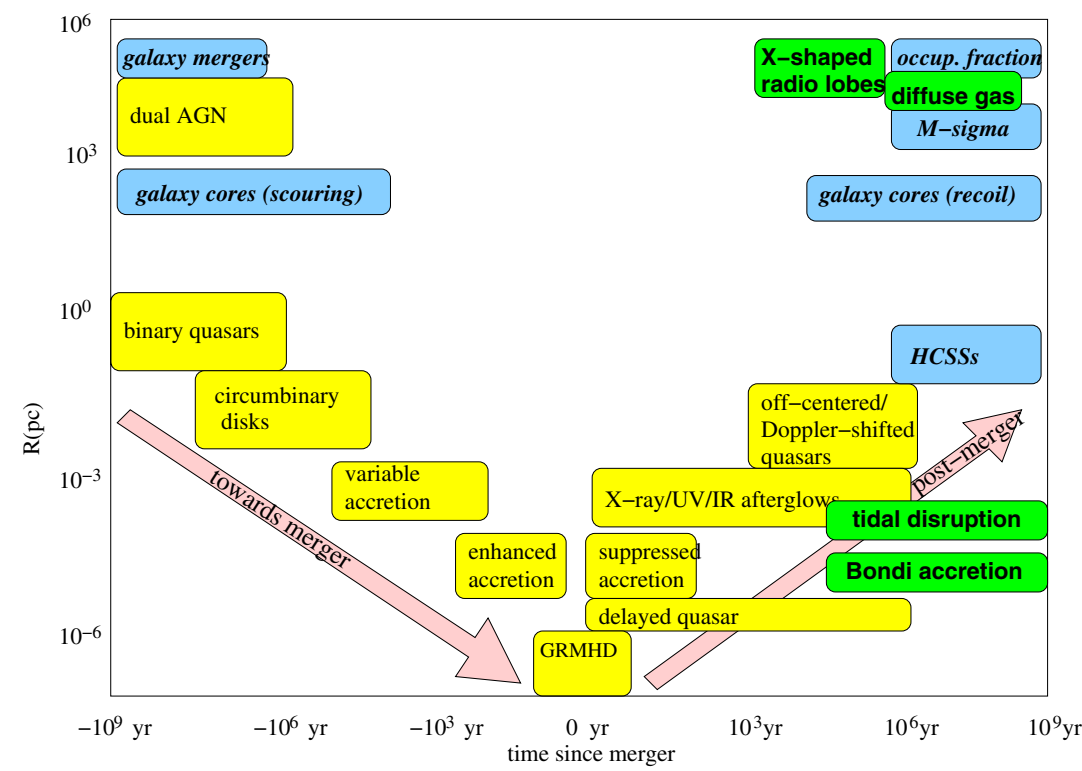

Figure 4. Selection of potential EM sources, sorted by timescale, typical size of emission region, and physical mechanism (blue/italic = stellar; yellow/times-roman = accretion disc; green/bold = diffuse gas/miscellaneous). The evolution of the merger proceeds from the upper-left through the lower-center, to the upper-right.

or even the fossil record of life on Earth, where a single consistent timeline of the evolution of various species can be reconstructed by matching together hundreds of independent, yet overlapping slices of geological strata. 


\subsection{Stellar signatures}

On the largest scales, we have strong circumstantial evidence of super-massive BH mergers at the centers of merging galaxies. From large optical surveys of interacting galaxies out to redshifts of $z \sim 1$, we can infer that $5-10 \%$ of massive galaxies are merging at any given time, and the majority of galaxies with $M_{\text {gal }} \gtrsim 10^{10} M_{\odot}$ have experienced a major merger in the past 3 Gyr [20, 42, 60, 162], with even higher merger rates at redshifts $z \sim 1-3$ [53]. At the same time, evidence was growing that the nucleus of nearly every large galaxy contained a central dark mass concentration [139, 161]. Yet we see a remarkably small number of dual AGN [52, 134], and only one known source with an actual binary system where the BHs are gravitationally bound to each other $[210,211]$. Taken together, these observations strongly suggest that when galaxies merge, the merger of their central SMBHs inevitably follows, and likely occurs on a relatively short time scale, which would explain the apparent scarcity of binary BHs (although recent estimates by [104] predict as many as $10 \%$ of AGNs with $M \sim 10^{7} M_{\odot}$ might be in close binaries with $a \sim 0.01 \mathrm{pc}$ ).

There is additional indirect evidence for SMBH mergers in the stellar distributions of galactic nuclei, with many elliptical galaxies showing light deficits (cores), which correlate strongly with the central BH mass [140]. The cores suggest a history of binary BHs that scour out the nuclear stars via three-body scattering $[169,171,172]$, or even post-merger relaxation of recoiling BHs [39, 92, 93, 167].

While essentially all massive nearby galaxies appear to host central SMBHs, it is quite possible that this is not the case at larger redshifts and smaller masses, where major mergers could lead to the complete ejection of the resulting black hole via large recoils. By measuring the occupation fraction of SMBHs in distant galaxies, one could infer merger rates and the distribution of kick velocities [218, 219, 264, 265, 267]. The occupation fraction will of course also affect the LISA event rates, especially at high redshift [227].

Another indirect signature of $\mathrm{BH}$ mergers comes from the population of stars that remain bound to a recoiling black hole that gets ejected from a galactic nucleus [135, 170, 183]. These stellar systems will appear similar to globular clusters, yet with smaller spatial extent and much larger velocity dispersions, as the potential is completely dominated by the central SMBH. With multi-object spectrometers on large ground-based telescopes, searching for these stellar clusters in the Milky Way halo or nearby galaxy clusters $(d \lesssim 40 \mathrm{Mpc})$ is technically realistic in the immediate future. These objects would also provide extremely strong evidence of black hole recoil, which is currently the only explanation for a stellar system with mass-to-light ratio of $>100 \times M_{\odot} / L_{\odot}$ and velocity dispersion of $>200 \mathrm{~km} \mathrm{~s}^{-1}[170]$.

\subsection{Gas signatures: accretion disks}

As discussed above in section 2, circumbinary discs will likely have a low-density gap within $r \approx 2 a$, although may still be able to maintain significant gas accretion across this gap, even forming individual accretion discs around each black hole. The most sophisticated GRMHD simulations suggest that this accretion can be maintained even as the binary is rapidly shrinking due to gravitational radiation [181]. If the inner discs can survive long enough, the final inspiral may lead to a rapid enhancement of accretion power as the fossil gas is plowed into the central black hole shortly before merger [5, 47]. For small values of $q$, a narrow gap could form in the inner disc, changing the AGN spectra in a potentially observable way [95, 163].

Regardless of how the gas reaches the central BH region, the simulations described above in section 3 all seem to agree that even a modest amount of magnetized gas can lead to a strong EM signature. If the primary energy source for heating the gas is gravitational [261], then 
typical efficiencies will be on the order of $\sim 1-10 \%$, comparable to that expected for standard accretion in AGN, although the much shorter timescales could easily lead to super-Eddington transients, depending on the optical depth and cooling mechanisms of the gas[141].

However, if the merging $\mathrm{BHs}$ are able to generate strong magnetic fields $[87,175,190,192]$, then hot electrons could easily generate strong synchrotron flux, or highly relativistic jets may be launched along the resulting BH spin axis, converting matter to energy with a Lorentz boost factor of $\Gamma \gg 1$. Even with purely hydrodynamic heating, particularly bright and long-lasting afterglows may be produced in the case of large recoil velocities, which effectively can disrupt the entire disc, leading to strong shocks and dissipation [3, 54, 149, 165, 215, 221, 241, 250, 271]. Long-lived afterglows could be discovered in existing multi-wavelength surveys, but successfully identifying them as merger remnants as opposed to obscured AGN or other bright unresolved sources would require improved pipeline analysis of literally millions of point sources, as well as extensive follow-up observations [221].

For many of these large-kick systems, we may observe quasar activity for millions of years after, with the source displaced from the galactic center, either spatially [51, 69, 121, $124,155,266]$ or spectroscopically [36, 38, 137, 209]. However, large offsets between the redshifts of quasar emission lines and their host galaxies have also been interpreted as evidence of pre-merger binary BHs $[34,65,68,254]$ or due to the large relative velocities in merging galaxies [59, 107, 242, 263], or 'simply' extreme examples of the class of double-peaked emitters, where the line offsets are generally attributed to the disc [50, 71, 84, 85, 243].

Thus to provide a strong argument for a relatively recent (within the past few Myr) SMBH merger, a single source would ideally present multiple pieces of circumstantial evidence, sharing the properties mentioned above: an offset AGN with offset emission lines consistent with the projected separation and time since merger, as further supported by the host galaxy morphology. A more indirect and admittedly ambiguous signature for kicked BHs could potentially show up in the statistical properties of active galaxies, in particular in the relative distribution of different classes of AGN in the 'unified model' paradigm $[28,136]$.

For systems that open up a gap in the circumbinary disc, another EM signature may take the form of a quasar suddenly turning on as the gas refills the gap, months to years after the BH merger [174, 236, 251]. But again, these sources would be difficult to distinguish from normal AGN variability without known GW counterparts. Some limited searches for this type of variability have recently been carried out in the X-ray band [122], but for large systematic searches, we will need targeted time-domain wide-field surveys like PTF, Pan-STARRS, and eventually LSST. One of the most valuable scientific products from these time-domain surveys will be a better understanding of what is the range of variability for normal AGN, which will help us distinguish when an EM signal is most likely due to a binary [253].

In addition to the many potential prompt and afterglow signals from merging BHs, there has also been a significant amount of theoretical and observational work focusing on the early precursors of mergers. Following the evolutionary trail in figure 1, we see that shortly after a galaxy merges, dual AGN may form with typical separations of a few kpc [52, 134], sinking to the center of the merged galaxy on a relatively short timescale $(\lesssim 1 \mathrm{Gyr})$ due to dynamical friction [18]. The galaxy merger process is also expected to funnel a great deal of gas to the galactic center, in turn triggering quasar activity [91, 108, 115, 125]. At separations of $\sim 1 \mathrm{pc}$, the BH binary (now 'hardened' into a gravitationally bound system) could stall, having depleted its loss cone of stellar scattering and not yet reached the point of gravitational radiation losses [173]. Gas dynamical drag from massive discs $\left(M_{\mathrm{disc}} \gg M_{\mathrm{BH}}\right)$ leads to a prompt inspiral ( $\sim 10 \mathrm{Myr})$, in most cases able to reach sub-parsec separations, depending on the resolution of the simulation $[55,64,66,67,73,74,126]$. 
At this point, a proper binary quasar is formed, with an orbital period of months to decades, which could be identified by periodic accretion [99, 100, 102, 160], density waves in the disc [103], or periodic red-shifted broad emission lines [33, 156, 177, 239]. If these binary AGN systems do in fact exist, spectroscopic surveys should be able to identify many candidates, which may then be confirmed or ruled out with subsequent observations over relatively short timescales $(\sim 1-10 \mathrm{yrs}$ ), as the line-of-site velocities to the BHs changes by an observable degree. This approach has been attempted with various initial spectroscopic surveys, but as yet, no objects have been confirmed to be binaries by multi-year spectroscopic monitoring $[38,50,72,146]$.

If such a source is eventually discovered, with blue/red-shifted emission lines that vary sinusoidally over a number of periods, it would arguably provide the strongest evidence to date of a merging SMBH binary. Furthermore, but measuring the detailed line profiles and offsets, the geometry, orientation, and masses of the binary could be well constrained.

\subsection{Gas signatures: diffuse gas; 'other'}

In addition to the many disc-related signatures, there are also a number of potential EM signatures that are caused by the accretion of diffuse gas in the galaxy. For the Poynting flux generated by the simulations of section 3 , transient bursts or modulated jets might be detected in all-sky radio surveys [123, 185]. For BHs that get significant kicks at the time of merger, we expect to see occasional episodes of Bondi accretion as the $\mathrm{BH}$ oscillates through the gravitational potential of the galaxy over millions of years, as well as off-center AGN activity [27, 82, 94, 244]. On larger spatial scales, the recoiling BH could also produce trails of over-density in the hot interstellar gas of elliptical galaxies [61]. Also on kpc-Mpc scales, $\mathrm{x}$-shaped radio jets have been seen in a number of galaxies, which could possibly be due to the merger and subsequent spin-flip of the central BHs [166].

Another potential source of EM counterparts comes not from diffuse gas, or accretion discs, but the occasional capture and tidal disruption of normal stars by the merging BHs. These tidal disruption events (TDEs), which also occurs in 'normal' galaxies [101, 133, 206], may be particularly easy to identify in off-center BHs following a large recoil [135]. TDE rates may be strongly increased immediately prior to the merger [48, 49, 222, 234, 248, 268], but the actual disruption signal may be truncated by the pre-merger binary [151], and postmerger recoil may also reduce the rates [148]. These TDE events are likely to be seen by the dozen in coming years with Pan-STARRS and LSST [86]. In addition to the tidal disruption scenario, in [222] we showed how gas or stars trapped at the stable Lagrange points in a BH binary could evolve during inspiral and eventually lead to enhanced star formation, ejected hyper-velocity stars, highly-shifted narrow emission lines, and short bursts of super-Eddington accretion coincident with the $\mathrm{BH}$ merger.

A completely different type of GW source can be observed with pulsar timing arrays (PTAs). In this technique, small time delays ( $\$ 10 \mathrm{~ns})$ in the arrival of pulses from millisecond radio pulsars would be direct evidence of extremely low-frequency (nano-Hertz) GWs from massive $\left(z 10^{8} M_{\odot}\right)$ BH binaries $[118,119,201,228-230,233,260]$. By cross-correlating the signals from multiple pulsars around the sky, we can effectively make use of a GW detector the size of the entire galaxy. For now, one of the main impediments to GW astronomy with pulsar timing is the relatively small number of known, stable millisecond radio pulsars. Current surveys are working to increase this number and the uniformity of their distribution on the sky [147].

Even conservative estimates suggest that PTAs are probably only about ten years away from a positive detection of the GW stochastic background signal from the ensemble of SMBH 
binaries throughout the universe [232]. The probability of resolving an individual source is significantly smaller, but if it were detected, would be close enough $(z \lesssim 1)$ to allow for extensive EM follow-up, unlike many of the expected LISA sources at $z \gtrsim 5$. Also, unlike LISA sources, PTA sources would be at an earlier stage in their inspiral and thus be much longer lived, allowing for even more extensive study. A sufficiently large sample of such sources would even allow us to test whether they are evolving due to GW emission or gasdriven migration [130, 231, 252] (a test that might also be done with LISA with only a single source with sufficient signal-to-noise [270]).

\section{Conclusion}

Black holes are fascinating objects. They push our intuition to the limits, and never cease to amaze us with their extreme behavior. For a high-energy theoretical astrophysicist, the only thing more exciting than a real astrophysical black hole is two black holes, destroying everything in their path as they spiral together toward the point of no return. Thus one can easily imagine the frustration that stems from our lack of ability to actually see such an event, despite the fact that it outshines the entire observable universe. Yet this challenge of direct detection provides motivation in at least equal measure to frustration. To paraphrase President Kennedy, we choose to search for merging black holes, not because they are easy to find, but because they are hard.

One important step along this path is the engagement of the broader (EM) astronomy community. Direct detection of GWs will not merely be a confirmation of a century-old theory — one more feather in Einstein's Indian chief head-dress-but the opening of a window through which we can observe the entire universe at once, eagerly listening for the next thing to go bang in the night. And when it does, all our EM eyes can swing over to watch the fireworks go off. With a tool as powerful as coordinated GW/EM observations, we will be able to answer many of the outstanding questions in astrophysics:

How were the first black holes formed? Where did the first quasars come from? What is the galaxy merger rate as a function of galaxy mass, mass ratio, gas fraction, cluster environment, and redshift? What is the mass function and spin distribution of the central BHs in these merging (and non-merging) galaxies? What is the central environment around the BHs, prior to merger: What is the quantity and quality (temperature, density, composition) of gas? What is the stellar distribution (age, mass function, metallicity)? What are the properties of the circumbinary disc? What is the time delay between galaxy merger and $\mathrm{BH}$ merger?

These are just a few of the mysteries that will be solved with the routine detection and characterization of SMBH mergers, may we witness them speedily in our days!

\section{Acknowledgments}

We acknowledge helpful conversations with John Baker, Manuela Campanelli, Bruno Giacomazzo, Bernard Kelly, Julian Krolik, Scott Noble, and Cole Miller.

\section{References}

[1] Abell P A et al 2009 arXiv:0912.0201

[2] Alic D, Moesta P, Rezzolla L, Zanotti O and Jaramillo J L 2012 Astrophys. J. 75436

[3] Anderson M, Lehner L, Megevand M and Neilsen D 2010 Phys. Rev. D 81044004

[4] Apostolatos T A, Cutler C, Sussman G J and Thorne K S 1994 Phys. Rev. D 496274

[5] Armitage P J and Natarajan P 2002 Astrophys. J. Lett. 567 9-12 
[6] Armitage P J and Natarajan P 2005 Astrophys. J. 634 921-7

[7] Arnowitt R, Deser S and Misner C W 2008 Gen. Rel. Grav. 40 1997-2007

[8] Artymowicz P, Clarke C J, Lubow S H and Pringle J E 1991 Astrophys. J. 370 L35-L38

[9] Artymowicz P and Lubow S H 1994 Astrophys. J. 421 651-67

[10] Artymowicz P and Lubow S H 1996 Astrophys. J. Lett. 46777

[11] Baker J G, Centrella J, Choi D-I, Koppitz M and van Meter J R 2006 Phys. Rev. Lett. 96111102

[12] Baker J G, Centrella J, Choi D-I, Koppitz M, van Meter J R and Miller M C 2006 Astrophys. J. Lett. 653 93-96

[13] Baker J G, Campanelli M, Pretorius F and Zlochower Y 2007 Class. Quantum Grav. 24 S25-S31

[14] Baker J G, Boggs W D, Centrella J, Kelly B J, McWilliams S T, Miller M C and van Meter J R 2007 Astrophys. J. $6681140-4$

[15] Balbus S A and Hawley J F 1998 Rev. Mod. Phys. 70 1-53

[16] Buonanno A and Damour T 1999 Phys. Rev. D 59084006

[17] Baruteau C, Ramirez-Ruiz E and Masset F 2012 Mon. Not. R. Astron. Soc. 423 L65

[18] Begelman M C, Blandford R D and Rees M J 1980 Nature 287 307-9

[19] Bekenstein J D 1973 Astrophys. J. 183657

[20] Bell E F et al 2006 Astrophys. J. $652270-6$

[21] Berti E, Cardoso V, Gonzalez J, Sperhake U, Hannam M, Husa S and Brugmann B 2007 Phys. Rev. D 76064034

[22] Berti E and Volonteri M 2008 Astrophys. J. 684822

[23] Berti E, Kesden M and Sperhake U 2012 Phys. Rev. D 85124049

[24] Blanchet L, Quasailah M S S and Will C M 2005 Astrophys. J. 635508

[25] Blanchet L 2006 Living Rev. Rel. 94

[26] Blandford R D and Znajek R L 1977 Mon. Not. R. Astron. Soc. 179433

[27] Blecha L and Loeb A 2008 Mon. Not. R. Astron. Soc. 390 1311-25

[28] Blecha L, Cox T J, Loeb A and Hernquist L 2011 Mon. Not. R. Astron. Soc. 412 2154-82

[29] Bloom J et al 2009 arXiv:0902.1527

[30] Bode T, Haas R, Bogdanovic T, Laguna P and Shoemaker D 2010 Astrophys. J. 7151117

[31] Bode T, Bogdanovic T, Haas R, Healy J, Laguna P and Shoemaker D 2010 Astrophys. J. 74445

[32] Bogdanovic T, Reynolds C S and Miller M C 2007 Astrophys. J. Lett. 661147

[33] Bogdanovic T, Smith B D, Sigurdsson S and Eracleous M 2008 Astrophys. J. Suppl. 174 455-80

[34] Bogdanovic T, Eracleous M and Sigurdsson S 2009 Astrophys. J. 697 288-92

[35] Bogdanovic T, Bode T, Haas R, Laguna P and Shoemaker D 2011 Class. Quantum Grav. 28094020

[36] Bonning E W, Shields G A and Salviander S 2007 Astrophys. J. Lett. 666 13-16

[37] Bonnor W B and Rotenberg M A 1961 Proc. R. Soc. A 2651320

[38] Boroson T A and Lauer T R 2009 Nature 458 53-55

[39] Boylan-Kolchin M, Ma C-P and Quataert E 2004 Astrophys. J. Lett. 613 37-40

[40] Boyle L and Kesden M 2008 Phys. Rev. D 78024017

[41] Boyle M, Brown D A, Kidder L E, Mroue A H, Pfeiffer H P, Scheel M A, Cook G P and Teukolsky S A 2007 Phys. Rev. D 76124038

[42] Bridge C R, Carlberg R G and Sullivan M 2010 Astrophys. J. 709 1067-82

[43] Campanelli M, Lousto C, Marronetti P and Zlochower Y 2006 Phys. Rev. Lett. 96111101

[44] Campanelli M, Lousto C and Zlochower Y 2006 Phys. Rev. D 74041501

[45] Campanelli M, Lousto C, Zlochower Y and Merritt D 2007 Astrophys. J. Lett. 659 5-8

[46] Centrella J, Baker J G, Kelly B J and van Meter J R 2010 Rev. Mod. Phys. 82 3069-119

[47] Chang P, Strubbe L E, Menou K and Quataert E 2010 Mon. Not. R. Astron. Soc. 407 2007-16

[48] Chen X, Madau P, Sesana A and Liu F K 2009 Astrophys. J. Lett. 697 149-52

[49] Chen X, Sesana A, Madau P and Liu F K 2011 Astrophys. J. 72913

[50] Chornock R, Bloom J S, Cenko S B, Filippenko A V, Silverman J M, Hicks M D, Lawrence K J, Mendez A J, Rafelski M and Wolfe A M 2010 Astrophys. J. Lett. 709 39-43

[51] Civano F et al 2010 Astrophys. J. 717 209-22

[52] Comerford J M et al 2009 Astrophys. J. 698 956-65

[53] Conselice C J, Bershady M A, Dickinson M and Papovich C 2003 Astron. J. 126 1183-207

[54] Corrales L R, Haiman Z and MacFadyen A 2010 Mon. Not. R. Astron. Soc. 404 947-62

[55] Cuadra J, Armitage P J, Alexander R D and Begelman M C 2009 Mon. Not. R. Astron. Soc. 393 1423-32

[56] Cutler C, Finn L S, Poisson E and Sussman G J 1993 Phys. Rev. D 47 1511-8

[57] Cutler C 1998 Phys. Rev. D 577089

[58] Damour T and Gopakumar A 2006 Phys. Rev. D 73124006

[59] Decarli R, Falomo R, Treves A and Barattini M 2010 Astron. Astrophys. 51127

[60] de Ravel L et al 2009 Astron. Astrophys. 498 379-97

[61] Devecchi B, Rasia E, Dotti M, Volonteri M and Colpi M 2009 Mon. Not. R. Astron. Soc. $394633-40$ 
[62] Dolence J C, Gammie C F, Moscibrodzka M and Leung P K 2009 Astrophys. J. Suppl. 184387

[63] D'Orazio D J, Haiman Z and MacFadyen A 2012 Mon. Not. R. Astron. Soc. submitted (arXiv:1210.0536)

[64] Dotti M, Colpi M, Haardt F and Mayer L 2007 Mon. Not. R. Astron. Soc. 379 956-62

[65] Dotti M, Montuori C, Decarli R, Volonteri M, Colpi M and Haardt F 2009 Mon. Not. R. Astron. Soc. 398 L $73-\mathrm{L} 77$

[66] Dotti M, Ruszkowski M, Paredi L, Colpi M, Volonteri M and Haardt F 2009 Mon. Not. R. Astron. Soc. 396 1640-6

[67] Dotti M, Volonteri M, Perego A, Colpi M, Ruszkowski M and Haardt F 2010 Mon. Not. R. Astron. Soc. $402682-90$

[68] Dotti M and Ruszkowski M 2010 Astrophys. J. Lett. 713 37-40

[69] Dottori H, Diaz R J, Albacete-Colombo J F and Mast D 2010 Astrophys. J. Lett. $71742-46$

[70] Elvis M et al 1978 Mon. Not. R. Astron. Soc. 183129

[71] Eracleous M, Halpern J P, Gilbert A M, Newman J A and Filippenko A V 1997 Astrophys. J. 490216

[72] Eracleous M, Boroson T A, Halpern J P and Liu J 2012 Astrophys. J. Suppl. 20123

[73] Escala A, Larson R B, Coppi P S and Mardones D 2004 Astrophys. J. 607 765-77

[74] Escala A, Larson R B, Coppi P S and Mardones D 2005 Astrophys. J. 630 152-66

[75] Estabrook F B and Wahlquist H D 1975 Gen. Rel. Grav. $6439-47$

[76] Faller J E, Bender P L, Hall J L, Hils D and Stebbins R T 1989 Adv. Space Res. 9 107-11

[77] Farris B D, Liu Y-K and Shapiro S L 2010 Phys. Rev. D 81084008

[78] Farris B D, Liu Y-K and Shapiro S L 2011 Phys. Rev. D 84024024

[79] Farris B D, Gold R, Pschalidis V, Etienne Z B and Shapiro S L 2012 Phys. Rev. Lett. 109221102

[80] Favata M, Hughes S A and Holz D E 2004 Astrophys. J. Lett. 6075

[81] Flanagan E E and Hughes S A 1998 Phys. Rev. D 574535

[82] Fujita Y 2009 Astrophys. J. 691 1050-7

[83] Gallouin L, Nakano H, Yunes N and Campanelli M 2012 Class. Quantum Grav. 29235013

[84] Gaskell M C 1988 Lect. Notes Phys. 30761

[85] Gaskell M C 2010 Nature 463 E1

[86] Gezari S et al 2009 Astrophys. J. 698 1367-79

[87] Giacomazzo B, Baker J G, Miller M C, Reynolds C S and van Meter J R 2012 Astrophys. J. Lett. 75215

[88] Goldreich P and Tremaine S 1979 Astrophys. J. 233 857-71

[89] Gonzalez J A, Hannam M, Sperhake U, Brugmann B and Husa S 2007 Phys. Rev. Lett. 98091101

[90] Gonzalez J A, Hannam M, Sperhake U, Brugmann B and Husa S 2007 Phys. Rev. Lett. 98231101

[91] Green P J, Myers A D, Barkhouse W A, Mulchaey J S, Bennert V N, Cox T J and Aldcroft T L 2010 Astrophys. J. $7101578-88$

[92] Gualandris A and Merritt D 2008 Astrophys. J. 678 780-97

[93] Guedes J, Madau P, Kuhlen M, Diemand J and Zemp M 2009 Astrophys. J. 702 890-900

[94] Guedes J, Madau P, Mayer L and Callegari S 2011 Astrophys. J. 729125

[95] Gultekin K and Miller J M 2012 Astrophys. J. 76190

[96] Gunther R and Kley W 2002 Astron. Astrophys. 387550

[97] Gunther R, Schafer C and Kley W 2004 Astron. Astrophys. 423559

[98] Hahn S G and Lindquist R W 1964 Ann. Phys. 29304

[99] Haiman Z, Kocsis B, Menou K, Lippai Z and Frei Z 2009 Class. Quantum Grav. 26094032

[100] Haiman Z, Kocsis B and Menou K 2009 Astrophys. J. 700 1952-69

[101] Halpern J P, Gezari S and Komossa S 2004 Astrophys. J. 604572

[102] Hayasaki K, Mineshige S and Ho L C 2008 Astrophys. J. 682 1134-40

[103] Hayasaki K and Okazaki A T 2009 Astrophys. J. Lett. 6915

[104] Hayasaki K, Ueda Y and Isobe N 2010 Publ. Astron. Soc. Japan 621351

[105] Hayasaki K 2011 Astrophys. J. Lett. 726145

[106] Hayasaki K, Saito H and Mineshige S 2012 Publ. Astron. Soc. Japan submitted (arXiv:1211.5137)

[107] Heckman T M, Krolik J H, Moran S M, Schnittman J D and Gezari S 2009 Astrophys. J. 695 363-7

[108] Hernquist L 1989 Nature 340687

[109] Herrmann F, Hinder I, Shoemaker D, Laguna P and Matzner R A 2007 Astrophys. J. 661 430-6

[110] Herrmann F, Hinder I, Shoemaker D and Laguna P 2007 Class. Quantum Grav. 24 S33

[111] Hinder I, Vaishnav B, Herrmann F, Shoemaker D M and Laguna P 2008 Phys. Rev. D 77081502

[112] Hinder I et al (for the NRAR Collaboration) 2013 arXiv: 1307.5307

[113] Hirose S, Krolik J H and Blaes O 2009 Astrophys. J. 69116

[114] Hirose S, Blaes O and Krolik J H 2009 Astrophys. J. 704 781-8

[115] Hopkins P F, Hernquist L, Cox T J and Keres D 2008 Astrophys. J. Suppl. 175356

[116] Ivanov P B, Papaloizou J C B and Polnarev A G 1999 Mon. Not. R. Astron. Soc. 30779 
[117] Jaramillo J L, Macedo R P, Moesta P and Rezzolla L 2012 Phys. Rev. D 85084030

[118] Jenet F A et al 2006 Astrophys. J. 653 1571-6

[119] Jenet F A et al 2009 arXiv:0909.1058

[120] Jiang Y-F, Stone J M and Davis S W 2012 Astrophys. J. Suppl. 19914

[121] Jonker P G, Torres M A P, Fabian A C, Heida M, Miniutti G and Pooley D 2010 Mon. Not. R. Astron. Soc. $407645-50$

[122] Kanner J, Baker J G, Blackburn L, Camp J, Mooley K, Mushotzky R and Ptak A 2013 Astrophys. J. submitted (arXiv:1305.5874)

[123] Kaplan D L, O’Shaugnessy R, Sesana A and Volonteri M 2011 Astrophys. J. Lett. 73437

[124] Kapoor R C 1976 Pramãna 7 334-43

[125] Kauffmann G and Haehnelt M 2000 Mon. Not. R. Astron. Soc. 311576

[126] Kazantzidis S, Mayer L, Colpi M, Madau P, Debattista V P, Wadsley J, Stadel J, Quinn T and Moore B 2005 Astrophys. J. Lett. 623 L67-L70

[127] Kidder L E 1995 Phys. Rev. D 52821

[128] Kocsis B, Frei Z, Haiman Z and Menou K 2006 Astrophys. J. 637 27-37

[129] Kocsis B, Haiman Z and Menou K 2008 Astrophys. J. 684 870-87

[130] Kocsis B and Sesana A 2011 Mon. Not. R. Astron. Soc. 4111467

[131] Kocsis B, Haiman Z and Loeb A 2012 Mon. Not. R. Astron. Soc. 4272660

[132] Kocsis B, Haiman Z and Loeb A 2012 Mon. Not. R. Astron. Soc. 4272680

[133] Komossa S and Bode N 1999 Astron. Astrophys. 343 775-87

[134] Komossa S, Burwitz V, Hasinger G, Predehl P, Kaastra J S and Ikebe Y 2003 Astrophys. J. Lett. 582 15-19

[135] Komossa S and Merritt D 2008 Astrophys. J. Lett. 683 21-24

[136] Komossa S and Merritt D 2008 Astrophys. J. Lett. 689 89-92

[137] Komossa S, Zhou H and Lu H 2008 Astrophys. J. Lett. 678 81-84

[138] Koppitz M, Pollney D, Reisswig C, Rezzolla L, Thornburg J, Diener P and Schnetter E 2007 Phys. Rev. Lett. 99041102

[139] Kormendy J and Richstone D 1995 Ann. Rev. Astron. Astrophys. 33581

[140] Kormendy J, Fisher D B, Cornell M E and Bender R 2009 Astrophys. J. Suppl. 182 216-309 Kormendy J and Bender R 2009 Astrophys. J. Lett. 691 142-6

[141] Krolik J H 2010 Astrophys. J. 709 774-9

[142] Lang R N and Hughes S A 2006 Phys. Rev. D 74122001

[143] Lang R N and Hughes S A 2008 Astrophys. J. 677 1184-200

[144] Lang R N and Hughes S A 2009 Class. Quantum Grav. 26094035

[145] Larwood J D and Papaloizou J C B 1997 Mon. Not. R. Astron. Soc. 285288

[146] Lauer T A and Boroson T R 2009 Astrophys. J. 703 930-8

[147] Lee K J et al 2013 Mon. Not. R. Astron. Soc. 433688

[148] Li S, Liu F K, Berczik P, Chen X and Sperzem R 2012 Astrophys. J. 74865

[149] Lippai Z, Frei Z and Haiman Z 2008 Astrophys. J. Lett. 676 5-8

[150] European Space Agency 2011 LISA Assessment Study Report ('Yellow Book') ESA/SRE(2011)3

[151] Liu F K, Li S and Chen X 2009 Astrophys. J. Lett. 706 133-7

[152] Liu Y T and Shapiro S L 2010 Phys. Rev. D 82123011

[153] Lodato G, Nayakshin S, King A R and Pringle J E 2009 Mon. Not. R. Astron. Soc. 3981392

[154] Lodato G and Gerosa D 2013 Mon. Not. R. Astron. Soc. Lett. 42930

[155] Loeb A 2007 Phys. Rev. Lett. 99041103

[156] Loeb A 2010 Phys. Rev. D 81047503

[157] Lousto C O and Zlochower Y 2009 Phys. Rev. D 79064018

[158] Lousto C O, Zlochower Y, Dotti M and Volonteri M 2012 Phys. Rev. D 85084015

[159] Lynden-Bell D 1969 Nature 223690

[160] MacFadyen A I and Milosavljevic M 2008 Astrophys. J. 672 83-93

[161] Magorrian J et al 1998 Astron. J. 115 2285-305

[162] McIntosh D H, Guo Y, Hertzberg J, Katz N, Mo H J, van den Bosch F C and Yang X 2008 Mon. Not. R. Astron. Soc. 388 1537-56

[163] McKernan B, Ford K E S, Kocsis B and Haiman Z 2013 Mon. Not. R. Astron. Soc. 4321468

[164] McWilliams S T, Thorpe J I, Baker J G and Kelly B J 2010 Phys. Rev. D 81064014

[165] Megevand M, Anderson M, Frank J, Hirschmann E W, Lehner L, Liebling S L, Motl P M and Neilsen D 2009 Phys. Rev. D 80024012

[166] Merrit D and Ekers R D 2002 Science 297 1310-3

[167] Merritt D, Milosavljevic M, Favata M, Hughes S A and Holz D E 2004 Astrophys. J. Lett. 607 9-12

[168] Merritt D and Milosavljevic M 2005 Living Rev. Rel. 88 
[169] Merritt D, Mikkola S and Szell A 2007 Astrophys. J. 671 53-72

[170] Merritt D, Schnittman J D and Komossa S 2009 Astrophys. J. 699 1690-710

[171] Milosavljevic M and Merritt D 2001 Astrophys. J. 563 34-62

[172] Milosavljevic M, Merritt D, Rest A and van den Bosch F C 2002 Mon. Not. R. Astron. Soc. 331 51-55

[173] Milosavljevic M and Merritt D 2003 Astrophys. J. 596 860-78

[174] Milosavljevic M and Phinney E S 2005 Astrophys. J. Lett. 622 93-96

[175] Moesta P, Palenzuela C, Rezzolla L, Lehner L, Yoshida S and Pollney D 2010 Phys. Rev. D 81064017

[176] Moesta P, Alic D, Rezzolla L, Zanotti O and Palenzuela C 2012 Astrophys. J. Lett. 74932

[177] Montuori C, Dotti M, Colpi M, Decarli R and Haardt F 2011 Mon. Not. R. Astron. Soc. 41226

[178] Murray S S 2012 SPIE 8443 1L

[179] Nixon C J, King A R and Pringle J E 2011 Mon. Not. R. Astron. Soc. Lett. 41766

[180] Nixon C J 2012 Mon. Not. R. Astron. Soc. 4232597

[181] Noble S C, Mundim B C, Nakano H, Krolik J H, Campanelli M, Zlochower Y and Yunes N 2012 Astrophys. J. 75551

[182] Novikov I D and Thorne K D 1973 Black Holes ed C DeWitt and B S DeWitt (New York: Gordon and Breach)

[183] O'Leary R M and Loeb A 2009 Mon. Not. R. Astron. Soc. 395 781-6

[184] O'Neill S M, Miller M C, Bogdanovic T, Reynolds C S and Schnittman J D 2009 Astrophys. J. 700 859-71

[185] O'Shaughnessy R, Kaplan D L, Sesana A and Kamble A 2011 Astrophys. J. 743136

[186] Oda M, Gorenstein P, Gursky H, Kellogg E, Schreier E, Tanenbaum H and Giacconi R 1971 Astrophys. J. Lett. 1661

[187] Ohsuga K and Mineshige S 2011 Astrophys. J. 7362

[188] Ostriker J P and Tremaine S D 1975 Astrophys. J. Lett. 202 113-7

[189] Ostriker J P and Hausman M A 1977 Astrophys. J. Lett. 217 125-9

[190] Palenzuela C, Anderson M, Lehner L, Liebling S L and Neilsen D 2009 Phys. Rev. Lett. 103081101

[191] Palenzuela C, Lehner L and Yoshida S 2010 Phys. Rev. D 81084007

[192] Palenzuela C, Garrett T, Lehner L and Liebling S L 2010 Phys. Rev. D 82044045

[193] Palenzuela C, Lehner L and Liebling S L 2010 Science 329927

[194] Pan Y, Buonanno A, Boyle M, Buchman L T, Kidder L E, Pfeiffer H P and Scheel M A 2011 Phys. Rev. D 84124052

[195] Peres A 1962 Phys. Rev. 1282471

[196] Peters P C and Mathews J 1963 Phys. Rev. 131 435-40

[197] Pnce M, Faber J A and Lombardi J C 2012 Astrophys. J. 74571

[198] Pretorius F 2005 Phys. Rev. Lett. 95121101

[199] Pretorius F 2007 arXiv:0710.1338

[200] Pringle J E 1991 Mon. Not. R. Astron. Soc. 248 754-9

[201] Pshirkov M S, Baskaran D and Postnov K A 2010 Mon. Not. R. Astron. Soc. 402 417-23

[202] Racine E, Buonanno A and Kidder L 2009 Phys. Rev. D 80044010

[203] Redmount I H and Rees M J 1989 Comment. Astrophys. 14 165-75

[204] Rafikov R R 2012 Astrophys. J. submitted (arXiv:1205.5017)

[205] Rees M J 1984 Ann. Rev. Astron. Astrophys. 22471

[206] Rees M J 1988 Nature 333 523-8

[207] Rezzolla L, Macedo R P and Jaramillo J L 2010 Phys. Rev. Lett. 104221101

[208] Rezzolla L 2013 arXiv:1303.6464

[209] Robinson A, Young S, Axon D J, Kharb P and Smith J E 2010 Astrophys. J. Lett. 717 123-6

[210] Rodriguez C, Taylor G B, Zavala R T, Peck A B, Pollack L K and Romani R W 2006 Astrophys. J. 646 49-60

[211] Rodriguez C, Taylor G B, Zavala R T, Pihlstrom Y M and Peck A B 2009 Astrophys. J. 69737

[212] Roedig C, Dotti M, Sesana A, Cuadra J and Colpi M 2011 Mon. Not. R. Astron. Soc. 4153033

[213] Roedig C, Sesana A, Dotti M, Cuadra J, Amaro-Seoane P and Haardt F 2012 Astron. Astrophys. 545 A127

[214] Rosotti G P, Lodato G and Price D J 2012 Mon. Not. R. Astron. Soc. 4251958

[215] Rossi E M, Lodato G, Armitage P J, Pringle J E and King A R 2010 Mon. Not. R. Astron. Soc. 401 2021-35

[216] Sadowski A, Narayan R, Tchekhovskoy A and Zhu Y 2013 Mon. Not. R. Astron. Soc. 4293533

[217] Salpeter E E 1964 Astrophys. J. 140796

[218] Schnittman J D and Buonanno A 2007 Astrophys. J. Lett. 662 63-66

[219] Schnittman J D 2007 Astrophys. J. Lett. 667 133-6

[220] Schnittman J D, Buonanno A, van Meter J R, Baker J G, Boggs W D, Centrella J, Kelly B J and McWilliams S T 2008 Phys. Rev. D 77044031

[221] Schnittman J D and Krolik J H Astrophys. J. 684 835-44

[222] Schnittman J D 2010 Astrophys. J. 72439

[223] Schnittman J D 2011 Class. Quantum Grav. 28094021 
[224] Schnittman J D, Krolik J H and Noble S C 2013 Astrophys. J. 769156

[225] Schnittman J D and Krolik J H 2013 Astrophys. J. 77711

[226] Schwarzschild K 1916 Prus. Acad. Sci. VII 189-96 (arXiv:physics/990503)

[227] Sesana A 2007 Mon. Not. R. Astron. Soc. Lett. 382 6-10

[228] Sesana A, Vecchio A and Colacino C N 2008 Mon. Not. R. Astron. Soc. 390 192-209

[229] Sesana A, Vecchio A and Volonteri M 2009 Mon. Not. R. Astron. Soc. 394 2255-65

[230] Sesana A and Vecchio A 2010 Phys. Rev. D 81104008

[231] Sesana A, Roedig C, Reynolds M T and Dotti M 2012 Mon. Not. R. Astron. Soc. 420860

[232] Sesana A 2013 Mon. Not. R. Astron. Soc. Lett. 4331

[233] Seto N 2009 Mon. Not. R. Astron. Soc. 400 L38-42

[234] Seto N and Muto T 2010 Phys. Rev. D 81103004

[235] Shakura N I and Sunyaev R A 1973 Astron. Astrophys. 24337

[236] Shapiro S L 2010 Phys. Rev. D 81024019

[237] Shapiro S L 2013 Phys. Rev. D 87103009

[238] Shcherbakov R V and Huang L 2011 Mon. Not. R. Astron. Soc. 4101052

[239] Shen Y and Loeb A 2010 Astrophys. J. 725249

[240] Shi J-M, Krolik J H, Lubow S H and Hawley J F 2012 Astrophys. J. 749118

[241] Shields G A and Bonning E W 2008 Astrophys. J. 682 758-66

[242] Shields G A, Bonning E W and Salviander S 2009 Astrophys. J. 696 1367-73

[243] Shields G A et al 2009 Astrophys. J. 707 936-41

[244] Sijacki D, Springel V and Haehnelt M 2011 Mon. Not. R. Astron. Soc. 4143656

[245] Silk J and White S D 1978 Astrophys. J. Lett. 223 59-62

[246] Spergel D et al 2013 arXiv:1305.5422

[247] Stone J M and Norman M L 1992 Astrophys. J. Suppl. $80753-90$

[248] Stone N and Loeb A 2011 Mon. Not. R. Astron. Soc. 41275

[249] Takeuchi T, Miyama S M and Lin D N C 1996 Astrophys. J. 460832

[250] Tanaka T and Menou K 2010 Astrophys. J. 714 404-22

[251] Tanaka T, Haiman Z and Menou K 2010 Astron. J. 140 642-51

[252] Tanaka T, Menou K and Haiman Z 2012 Mon. Not. R. Astron. Soc. 420705

[253] Tanaka T 2013 Mon. Not. R. Astron. Soc. at press (arXiv:1303.6279)

[254] Tang S and Grindlay J 2009 Astrophys. J. 704 1189-94

[255] Thorpe J I, McWilliams S T, Kelly B J, Fahey R P, Arnaud K and Baker J G 2009 Class. Quantum Grav. 26094026

[256] Thorne K S and Braginsky V B 1976 Astrophys. J. Lett. 204 1-6

[257] Thorne K S 1994 Black Holes and Time Warps: Einstein's Outrageous Legacy (New York: W W Norton)

[258] Tichy W and Marronetti P 2007 Phys. Rev. D 76061502

[259] Toomre A 1977 Evolution of Galaxies and Stellar Populations: Proceedings of a Conference at Yale University ed B M Tinsley and R B Larson (New Haven: Yale University Observatory) p 401

[260] van Haasteren R and Levin Y 2010 Mon. Not. R. Astron. Soc. 401 2372-8

[261] van Meter J R, Wise J H, Miller MC, Reynolds C S, Centrella J, Baker J G, Boggs W D, Kelly B J and McWilliams S T 2010 Astrophys. J. Lett. 711 89-92

[262] van Meter J R, Miller M C, Baker J G, Boggs W D and Kelly B J 2010 Astrophys. J. Lett. 719 1427-32

[263] Vivek M, Srianand R, Noterdaeme P, Mohan V and Kuriakosde V C 2009 Mon. Not. R. Astron. Soc. 400 L6-L9

[264] Volonteri M 2007 Astrophys. J. Lett. 663 5-8

[265] Volonteri M, Lodato G and Natarajan P 2008 Mon. Not. R. Astron. Soc. 383 1079-88

[266] Volonteri M and Madau P 2008 Astrophys. J. Lett. 687 57-60

[267] Volonteri M, Gultekin K and Dotti M 2010 Mon. Not. R. Astron. Soc. 404 2143-50

[268] Wegg C and Bode N J 2011 Astrophys. J. Lett. 7388

[269] Wiseman A G 1992 Phys. Rev. D 461517

[270] Yunes N, Kocsis B, Loeb A and Haiman Z 2011 Phys. Rev. Lett. 107171103

[271] Zanotti O, Rezzolla L, Del Zanna L and Palenzuela C 2010 Astron. Astrophys. 5238

[272] Zanotti O 2013 New Astron. 17331 